\title{
Hemolysis Prediction by using a Power-Law Mathematical Model in an Aortic Arterial Section
}

\author{
M. Vílchez-Monge, M.Sc ${ }^{1}$, G. Ortiz-León, M.Sc ${ }^{1}$, and JM. Barboza-Retana, Lic ${ }^{1}$ \\ ${ }^{1}$ Instituto Tecnológico de Costa Rica, Costa Rica,mvilchez@tec.ac.cr,gaby@tec.ac.cr,jmbarboza@tec.ac.cr
}

\begin{abstract}
This paper describes the development of a method to calculate mechanical hemolysis experimented by red blood cells (RBCs) in the aortic arterial section. A model to obtain the rheological behavior of the blood in the aortic section is developed using COMSOL Multiphysics ${ }^{\circledR}$ and the RBCs trajectories are calculated using the Particle Tracing Module of COMSOL. The Blood model used is based on previous related works. The shear stress supported by the RBCs along the particle trajectories is used to calculate the hemolysis estimation using Matlab ${ }^{\circledR}$. For this, a power-law mathematical model is used to formulate an evaluation of the functional relationship between the shear stress and the hemolysis index. This formulation considers the effect of mechanical loading history experimented by the RBCs along his trajectories. A study of four RBCs is presented and here is included the results of trajectories, velocity profiles, shear stress and hemolysis index. From the results is observed that small shear stress values do not produce significant RBC damage, however if these values increases, the damage rises faster.
\end{abstract}

Keywords - red blood cells (RBCs); erythrocytes, mathematical model; mechanical hemolysis, shear stress.

Digital Object Identifier

(DOI):http://dx.doi.org/10.18687/LACCEI2016.1.1.116

ISBN: 978-0-9822896-9-3

ISSN: 2414-6390

$14^{\text {th }}$ LACCEI International Multi-Conference for Engineering, Education, and Technology: "Engineering Innovations for Global Sustainability", 20-22 July 2016, San José, Costa Rica. 


\title{
Hemolysis Prediction by using a Power-Law Mathematical Model in an Aortic Arterial Section
}

\author{
M. Vílchez-Monge, M.Sc ${ }^{1}$, G. Ortiz-León, M.Sc ${ }^{1}$, and JM. Barboza-Retana, Lic ${ }^{1}$ \\ ${ }^{1}$ Instituto Tecnológico de Costa Rica, Costa Rica, mvilchez@tec.ac.cr, gaby@tec.ac.cr,jmbarboza@tec.ac.cr
}

\begin{abstract}
This paper describes the development of a method to calculate mechanical hemolysis experimented by red blood cells (RBCs) in the aortic arterial section. A model to obtain the rheological behavior of the blood in the aortic section is developed

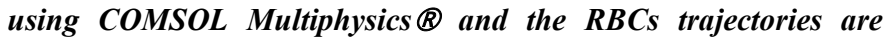
calculated using the Particle Tracing Module of COMSOL. The Blood model used is based on previous related works. The shear stress supported by the RBCs along the particle trajectories is used

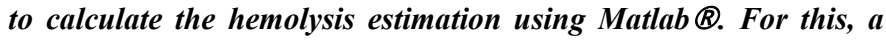
power-law mathematical model is used to formulate an evaluation of the functional relationship between the shear stress and the hemolysis index. This formulation considers the effect of mechanical loading history experimented by the RBCs along his trajectories. A study of four RBCs is presented and here is included the results of trajectories, velocity profiles, shear stress and hemolysis index. From the results is observed that small shear stress values do not produce significant RBC damage, however if these values increases, the damage rises faster.
\end{abstract}

Keywords - red blood cells (RBCs); erythrocytes, mathematical model; mechanical hemolysis, shear stress.

\section{INTRODUCTION}

Blood is a specialized connective tissue in which cells are suspended in an extracellular fluid called plasma. The so-called formed elements circulating in the plasma are erythrocytes (red blood cells), leukocytes (white blood cells) and platelets [4]. Red blood cells are the $99.9 \%$ of the cellular elements of blood [5]. They are differentiated structures lacking nucleus and completely filled with $\mathrm{O}_{2}$-carrying protein hemoglobin [4]. RBCs behave as neutrally buoyant microcapsules with high deformability but small superficial stretchability [6]. They have approximately $7.5 \mu \mathrm{m}$ in diameter, $2.6 \mu \mathrm{m}$ thick at the rim, but only $0.75 \mu \mathrm{m}$ thick in the center [4]. RBC membrane has a relaxation time of approximately $200 \mathrm{~ms}$ which depend on the age of the cell [7] and it can resist an estimated superficial strain of $6 \%$ before rupture [6]. In medical devices there are severe risk factors related to blood trauma: hemolysis (RBCs damage) and thrombosis (blood aggregation). The hemolysis is the hemoglobin release from RBCs by mechanical damage [8]. Hemolysis starts when the RBC membrane stretches, developing holes, and leaks hemoglobin [6]. There are different mathematical models to analyze hemolysis that are used in the design and evaluation of medical devices. These models should describe the sustained blood damage by RBCs when they are under unsteady stress conditions, taking into account the load history. Computational fluid dynamics (CFD) is an important design tool to analyze the blood damage; because allows to determinate velocity profiles, shear stress, recirculation and stagnation zones of the blood flow [9].

This work presents the implementation of a mathematical formulation of Power-Law model for calculate the hemolysis index in the aortic arterial section.

\section{FORMULATION OF POWER-LAW MATHEMATICAL MODEL}

A Power-law mathematical model is used to formulate an evaluation of the functional relationship between the concentration of hemoglobin released by damaged RBCs and the shear stress acting on blood as function of the exposure time, known as the hemolysis index $I(\%)$ [10]:

$$
I(\%)=\left(\frac{\Delta H b}{H b}\right)(\%)=C t^{a} \tau^{b}
$$

Where $C, b$ and $a$ are constant values obtained by experimental results, $\frac{\Delta H b}{H b}$ is the ratio of hemoglobin release in the plasma to the total hemoglobin, $\tau$ is the scalar measure of the shear stress acting on the blood in $P a$, and $t$ is the exposure time to the shear stress $\tau$ in seconds [8]. However, this model lacks sensitivity with respect to the cumulative effect of previously applied stress magnitudes [3].

Hemolysis prediction with mathematical models must fulfill 3 conditions proposed in [3]:

- Condition 1: It must not have a reduction of damage by a decreasing shear stress [11].

- Condition 2: When the mechanical load is constant, the result must be the same as with (1).

- Condition 3: Take account the load history of the RBCs, different load histories must have different blood damage results.

An alternative formulation of power-law model was proposed by Grigioni et al. in [3], where it is defined a mechanical quantity to describe the blood damage sustained by RBCs under unsteady stress conditions, taking into account the load history. This new formulation of the power-law model for blood damage prediction was originated from the observation of the similarities in aims and methods between the problem under investigation and dosimetry [3]. From (1), the basic formulation, it was defined a mechanical dose $D$ as:

$$
D=t \cdot \tau^{b / a}
$$

Digital Object Identifier (DOI): http://dx.doi.org/10.18687/LACCEI2016.1.1.116 ISBN: 978-0-9822896-9-3

ISSN: 2414-6390

14 ${ }^{\text {th }}$ LACCEI International Multi-Conference for Engineering, Education, and Technology: "Engineering Innovations for Global Sustainability”, 20-22 July 2016, San José, Costa Rica. 
Where Blood Damage index (BDI) is introduced from (1) and (2) as:

$$
B D I(\%)=C \cdot D^{a}
$$
[3]:

In relation with the above, two hypotheses were proposed in

- A relation between the accumulation of the blood damage and dose $D$.

- $D$ increases with the time.

Using the chain rule, $D$ can be expressed in differential form as:

$$
\frac{d D}{d t}=\frac{\partial D}{\partial t}+\frac{\partial D}{\partial \tau} \cdot \frac{d \tau}{d t}
$$

Assuming that during an infinitesimal time interval $d t$ (interval $\left.I_{a}=[t-d t, t]\right)$ the shear stress $\tau\left(t^{*}\right)\left(t^{*} \in I_{a}\right)$ is constant, the total derivate of (2) can be written as:

$$
d D=\tau^{b / a} d t
$$

From the integration with respect to the time of the two members of (5), the mechanical dose can be written as:

$$
D(t)-D\left(t_{0}\right)=\int_{t_{0}}^{t} \tau(\phi)^{b / a} d \phi
$$

Where $\tau$ is a function of time, and $D\left(t_{0}\right)$ is the initial value of the mechanical dose. The value of one infinitesimal interval of blood damage can be estimated from (3) as:

$$
d(B D I)=d\left(C D^{a}\right)=C d\left(D^{a}\right)=C a D^{a-1} d D
$$

Using (5) and (6), equation (7) can be written as:

$$
d(D B I)=C a\left(\int_{t_{0}}^{t} \tau(\xi)^{b / a} d \xi+D\left(t_{0}\right)\right)^{a-1} \tau(t)^{b / a} d t
$$

Where $t_{0}$ represents the initial time of the interval. The BDI can be now expressed as the integral sum of the infinitesimal contributions represented by (8):

$$
B D I=\int_{t_{0}}^{t} C a\left[\int_{t_{0}}^{\phi} \tau(\xi)^{b / a} d \xi+D\left(t_{0}\right)\right]^{a-1} \tau(\phi)^{b / a} d \phi
$$

The integral sum inside of square brackets in Eq. (9) represents the complete dose sustained by the RBC moving along a generic fluid pathline. Equation (8) can be expressed in his discrete form as:

$$
\Delta(B D I)_{i}=C a\left[\sum_{j=1}^{i} \tau\left(t_{j}\right)^{b / a} \Delta t_{j}+D\left(t_{0}\right)\right]^{a-1} \tau\left(t_{i}\right)^{b / a} \Delta t_{i}(10)
$$

Where $\Delta(B D I)_{i}$ quantifies the blood damage sustained by a $\mathrm{RBC}$ in the $i$ th interval (from instant $t_{i-1}$ to instant $t_{i}$ : this is the time interval in which is considered the shear stress constant); $t_{0}$ is the initial time interval; $\tau\left(t_{j}\right)$ is the shear stress value acting during the $j$ th observation time interval $\Delta t_{j}$; and $t_{j}$ is the time it takes the blood particle to reach the $j$ th observation point. From (10), it is possible to express the discrete form of (9) as:

$$
B D I=\sum_{i=1}^{N} C a\left[\sum_{j=1}^{i} \tau\left(t_{j}\right)^{b / a} \Delta t_{j}+D\left(t_{0}\right)\right]^{a-1} \tau\left(t_{i}\right)^{b / a} \Delta t_{i}
$$

Where $N$ is the number of observation time intervals along the fluid pathline. The term into the square brackets in (11) represents the whole mechanical dose acting on the erythrocyte when it moves along the pathline from the initial observation time until the $i$ th instant (conventionally is assumed $t_{0}=0$ ), while $\left(\tau\left(t_{i}\right)^{b / a} \Delta t_{i}\right)$ is the mechanical dose sustained by a RBC in the $i$ th interval, (from instant $t_{i-1}$ until instant $t_{i}$ ). Equation (11) states that the $i$ th experienced dose (the term inside the square brackets) is a weighted summation on the observation time intervals $\Delta t_{j}$ coming before time $i(j=1, \ldots, i)$, the $j$ th weight is the value of shear stress $\tau\left(t_{j}\right)$ sustained during the $j$ th time interval elevated to $b / a$ value.

In this work, the values used for parameters $\mathrm{a}, \mathrm{b}$ and $\mathrm{C}$ are [3] [10]:

$$
\text { - } \mathrm{a}=0.785, \mathrm{~b}=2.416, \mathrm{C}=3.62 \times 10^{-5}
$$

\section{SOLUTION}

The estimation of the hemolysis was done using a multiphysics simulation and mathematical formulation as post processing of the multiphysics results. First, COMSOL Multiphysics ${ }^{\circledR}$ was used to calculate the RBC trajectories and obtain the shear stress values along the aortic arterial section. This section was modeled with an approximated geometry that includes the ascending aorta, aortic arch, descending thoracic aorta and three bifurcations: brachiocephalic trunk, left carotid artery and left subclavian artery. Fig. 1 shows the model geometry.

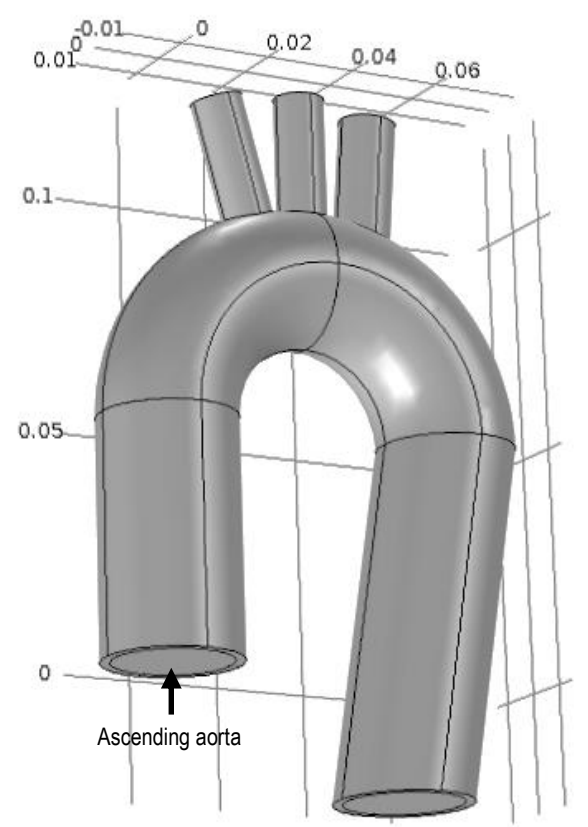

Fig. 1. Approximated geometry of the aortic arterial section (unit scale: $\mathrm{m}$ )

$14^{\text {th }}$ LACCEI International Multi-Conference for Engineering, Education, and Technology: "Engineering Innovations for 
Fluid-Structure Interaction module was used to simulate the interaction between the blood and blood vessel. A blood flow wave was used as input condition in the ascending aorta [1]. Fig. 2 shows this blood flow wave used in the simulation model. This wave shows the beginning of the blood ejection at $0.1 \mathrm{~s}$ and then the velocity rise to $150 \mathrm{~cm} / \mathrm{s}$ at $0.2 \mathrm{~s}$. Last, the closure of the aortic valve starts and a blood reflow is shown between $0.38 s$ and $0.49 s$.

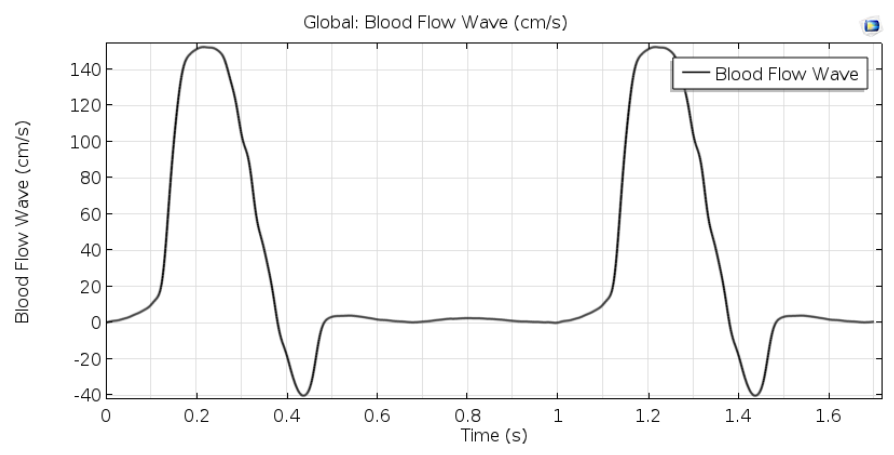

Fig. 2. Blood flow wave at the ascending aorta input

Particle tracing module was used to calculate the RBC trajectories along the aortic arterial section. RBCs were represented as spheres with a diameter of $7 \mu \mathrm{m}$ and a density of $1052,82 \mathrm{~kg} / \mathrm{m}^{\wedge} 3$. Finally, the formulation described previously, was implemented to estimate the damage received by the RBCs under analysis was used to obtain the BDI with a time interval $\Delta t$ of $1 \mathrm{~ms}$. Fig. 3 shows the algorithm to calculate the $B D I$ values to the RBCs in the analysis. Live Link for Matlab module was used to export this results from COMSOL Multiphysics ${ }^{\circledR}$ to Matlab ${ }^{\circledR}$ in order to calculate the $B D I$.

\section{RESULTS}

Simulation results from COMSOL Multiphysics ${ }^{\circledR}$ show the blood behaviour as a result of the boundary conditions and material properties. Fig. 4 shows the streamlines within the aortic arterial section at $0.2 \mathrm{~s}$, where there are not recirculation zones and the maximum velocity value is $2.09 \mathrm{~m} / \mathrm{s}$.

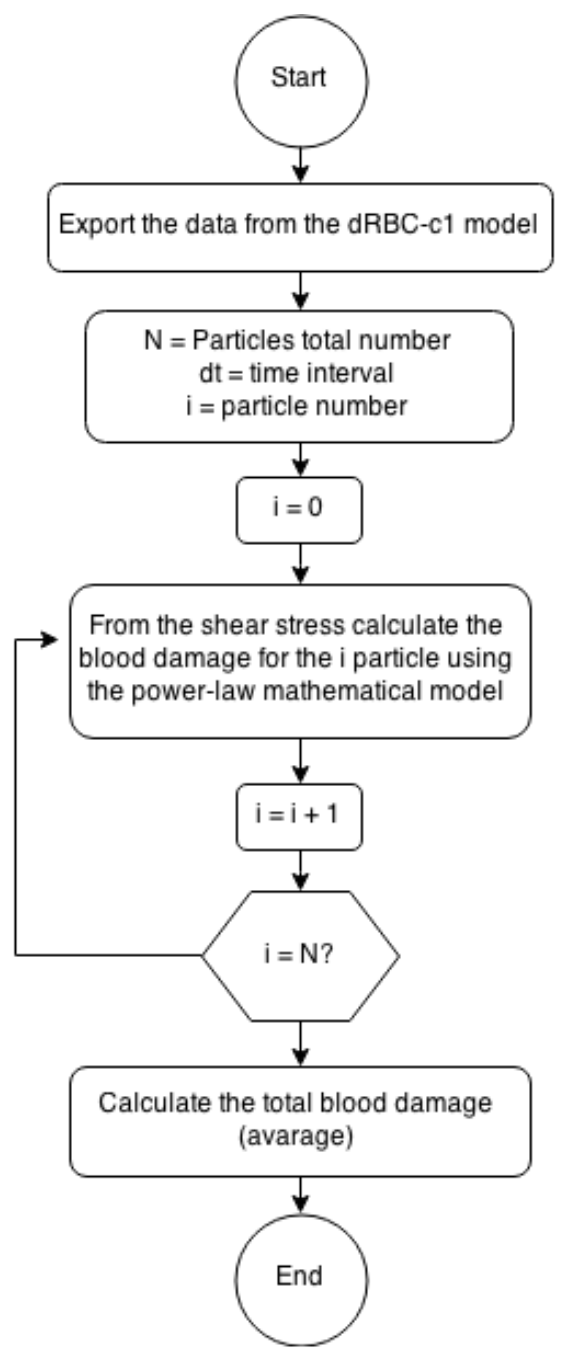

Fig. 3. Algorithm to calculate the hemolysis using the powerlaw formulation

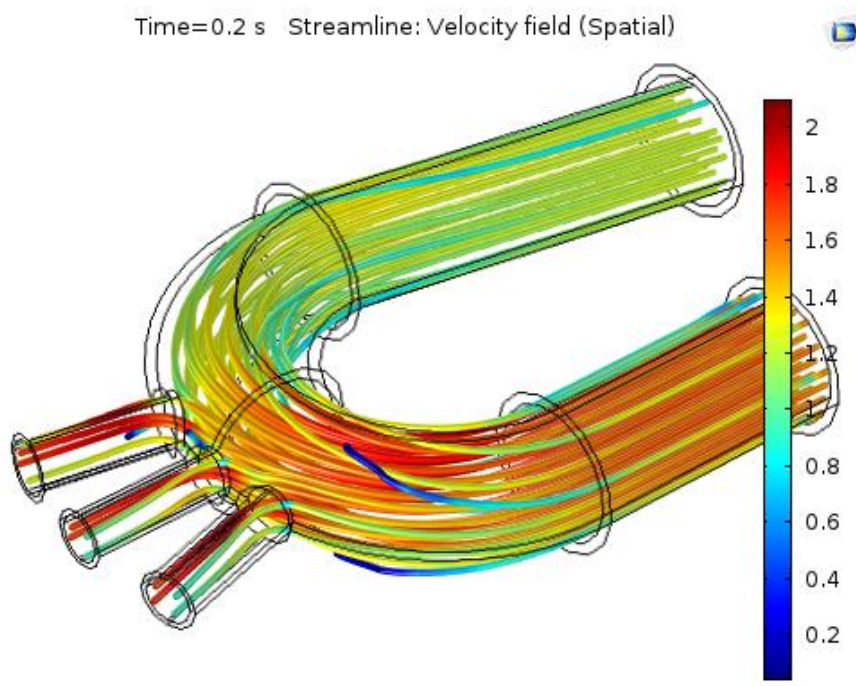

Fig. 4. Streamlines within the aortic arterial section $(\mathrm{m} / \mathrm{s})$

14 ${ }^{\text {th }}$ LACCEI International Multi-Conference for Engineering, Education, and Technology: "Engineering Innovations for 
Fig. 5 shows four RBC trajectories along the aortic arterial section. The color scale represents the particle velocity magnitude, where the maximum and minimum values were $196.90 \mathrm{~cm} / \mathrm{s}$ and $0.41 \mathrm{~cm} / \mathrm{s}$.

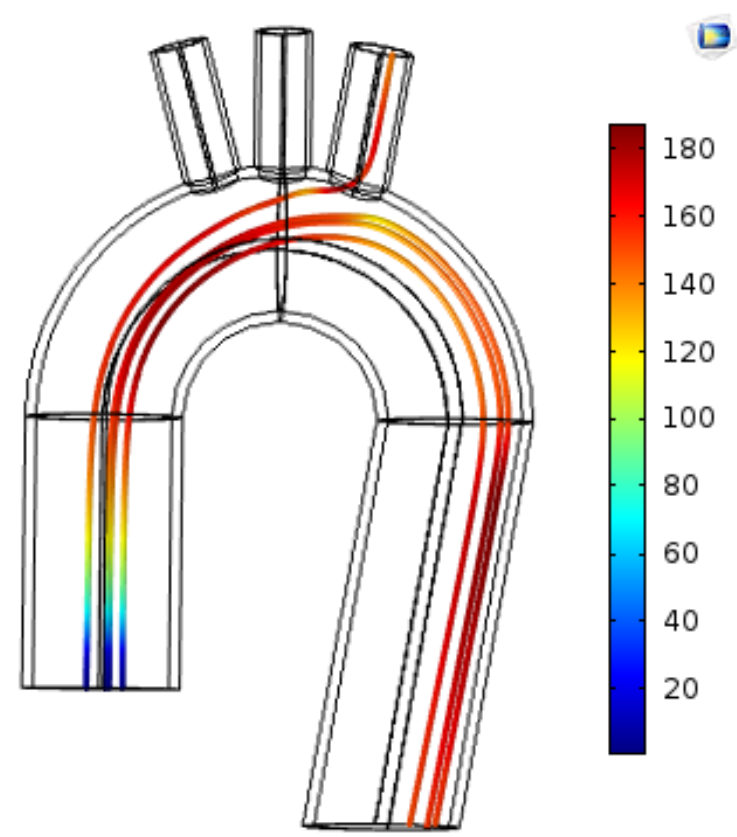

Fig. 5. Velocity magnitude along the trajectory particles $(\mathrm{cm} / \mathrm{s})$

Fig. 6 shows the shear rate along of the RBC trajectories. The maximum and minimum values were $344.69(1 / s)$ and $0.01(1 / s)$. High shear rate values were obtained when there are changes in the RBC trajectory direction.

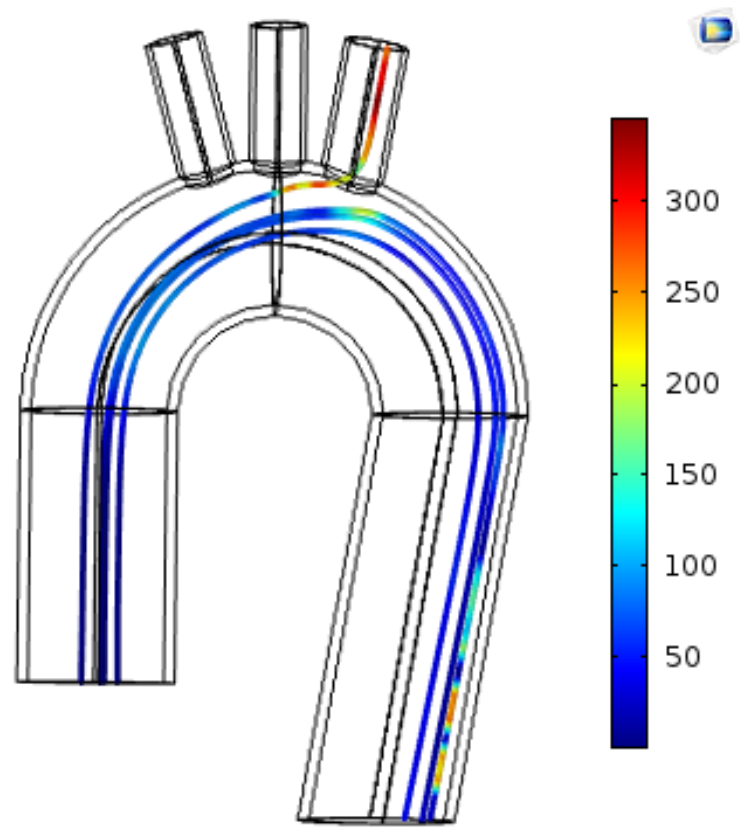

Fig. 6. Shear rate magnitude along the trajectory particles $(1 / \mathrm{s})$
The shear stress was calculated from the shear rate using the dynamic viscosity as:

$$
\tau=\mu * \dot{\gamma}
$$

Where, $\tau$ is the shear stress $\left(N / \mathrm{m}^{2}\right), \mu$ the dynamic viscosity $(\mathrm{mPa} \cdot \mathrm{s})$ and $\dot{\gamma}$ the shear rate $(1 / \mathrm{s})$. One of the four shear stress histories of the RBCs is shown in Fig. 7. It is observed that the BDI of the RBC increased with time (cumulative effect). Due to the mathematical nature of (11) (multiplication of factors), there is an attenuation of the dBDI values when the shear stress decrease or otherwise. In general, an increase or decrease of shear stress values by some factor produce a notable effect on the dBDI. The above is noted in Fig. 7 , where is show the attenuation of the dBDI values in the regions where the shear stress is lower. So, if the shear stress value increases by some external factor, dBDI will present a strong increase (for example in implantable devices).
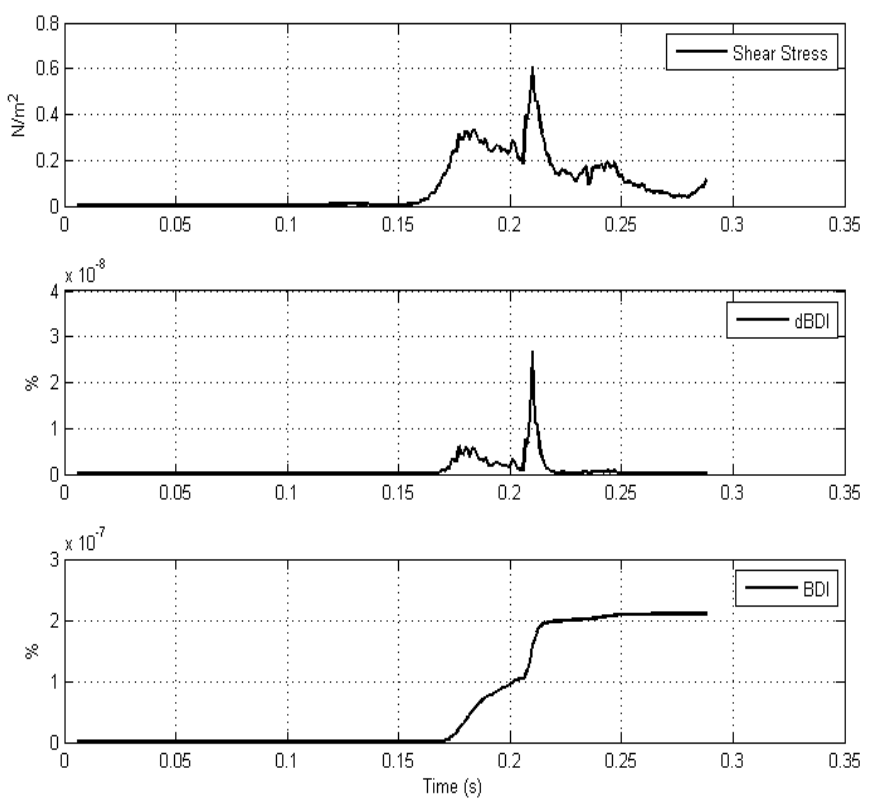

Fig. 7. Shear stress (top), differential blood damage (middle) and blood damage index (bottom) experimented by a RBC

Table 1 has the maximum values of shear stress and dBDI for all red blood cells in study. In the last column is shown the BDI final value.

Table 1. Blood damage results from the analysis of four RBC

\begin{tabular}{|c|c|c|c|}
\hline RBC & $\begin{array}{l}\text { Maximum Shear } \\
\text { Stress (N/m^2) }\end{array}$ & $\begin{array}{c}\text { Maximum } \\
\text { dBDI (\%) }\end{array}$ & $\begin{array}{c}\text { BDI } \\
\mathbf{( \% )}\end{array}$ \\
\hline 1 & 0.377 & $0.91 \times 10^{-8}$ & $1.44 \times 10^{-7}$ \\
\hline 2 & 0.611 & $2.71 \times 10^{-8}$ & $2.14 \times 10^{-7}$ \\
\hline 3 & 0.964 & $7.11 \times 10^{-8}$ & $10.11 \times 10^{-7}$ \\
\hline 4 & 1.205 & $12.82 \times 10^{-8}$ & $18.07 \times 10^{-7}$ \\
\hline
\end{tabular}


Hemolysis index estimation was calculated as a contribution of all RBCs in analysis, thereby the average dBDI and BDI results is shown in Fig. 8.
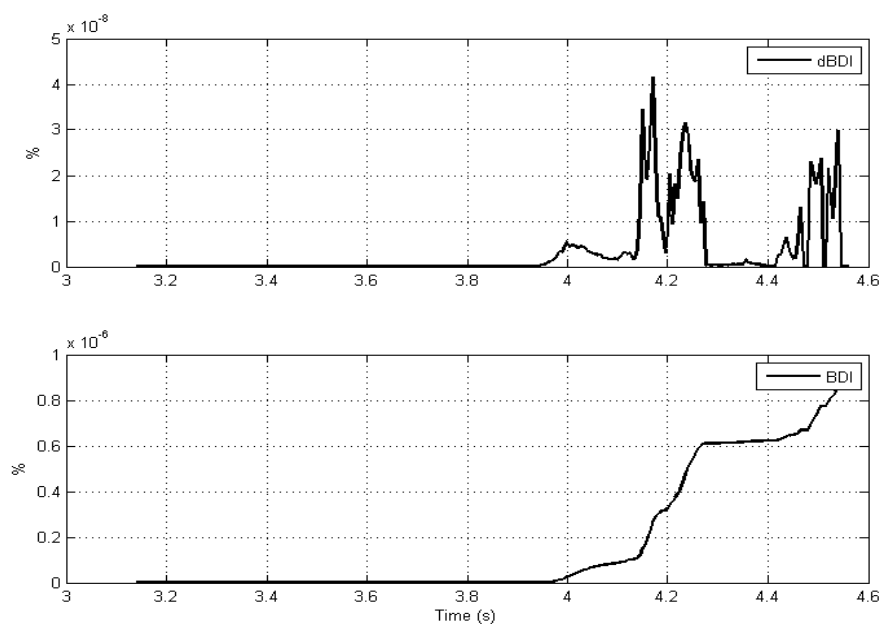

Fig. 8. Differential blood damage index and blood damage index as contribution of all red blood cells in analysis (average damage)

\section{CONCLUSIONS}

In this work, a blood damage model was analyzed and implemented to calculate hemolysis in the aortic arterial section. This model takes into account the load history, shear stress conditions and exposure time parameters to calculate the BDI experimented by a RBC. From the results was found a continuous increase of the BDI, according to the initial hypothesis (Condition 0). There are not similar models or experimental results (geometry, mathematical model, initial conditions, etc.) to compare these results with this work. In future works, it will be necessary to determine the minimum number of RBCs to get a good estimate of mechanical hemolysis with the BDI, because computing power and simulation time increase when more particles are included. Mechanical damage is the main cause of hemolysis in extracorporeal systems and implantable devices [3]. To date, different models have been developed to relate the flow conditions with the hemolysis. These models allow the identification of blood trauma regions in the design and evaluation of medical devices. Actually, CFD has been widely applied as tool in the blood damage prediction for implantable devices [6] [8] [9]. To finish: "These methods to quantify the blood trauma induced by artificial organs or implantable devices would reduce efforts and costs of design and development, and could be helpful in the assessments of clinical working conditions to minimize the impact of medical devices on blood cells" [3].

\section{ACKNOWLEDGMENT}

We thank the Office of the Vice Chancellor for Research and Extension (VIE) of the Costa Rica Institute of Technology, for supporting and providing the funds for the development of research project number 5401-1360-3801, to which this paper belongs and for providing the scholarship for postgraduate special research assistant student to author José Miguel Barboza according to contract $\mathrm{N}^{\mathrm{o}} 008-2015$.

[9] K. H. Fraser, M. E. Taskin, B. P. Griffith, and Z. J. $\mathrm{Wu}$, "The use of computacional fluid dynamics in the development of ventricular assist devices," Med Eng Phys, vol. 3, no. 33, pp. 263-280, 2011.

[10] M. Giersiepen, L. J. Wurzinger, R. Opitz, and H. Reul, "Estimation of shear stress-related blood damage in heart valve prostheses--in vitro comparison of 25 aortic valves.," Int. J. Artif. Organs, vol. 13, no. 5, pp. 300-6, May 1990.

[11] M. Grigioni, C. Daniele, U. Morbiducci, G. D’Avenio, G. Di Benedetto, and V. Barbaro, "The power-law mathematical model for blood damage prediction: analytical developments and physical inconsistencies.," Artif. Organs, vol. 28, no. 5, pp. 467-75, May 2004. 